\title{
Subsampling OFDM-based Ultrasonic Data Communication through Metallic Channels for Monitoring of Cargo Containers
}

\author{
José R. García Oya, José M. Algueta Miguel, José García Doblado, Fernando Muñoz Chavero, \\ Member, IEEE, Eduardo Hidalgo Fort, Vicente Baena Lecuyer, Member, IEEE, Antonio J. López \\ Martín, Senior Member, IEEE
}

\begin{abstract}
An enhanced ultrasonic communication system based on piezoelectric transducers for monitoring of goods in cargo containers is presented. The proposed system consists of several sensors placed inside the container, whose data are collected and transmitted outside it. Data transmission is carried out by an ultrasonic communication channel, in order to avoid drilling the wall of the container. The proposed data communication system is based on the transmission of a 128OFDM signal. This modulation has been chosen due to its robustness to channels with frequency-selective fading and its spectrum efficiency. In order to increase the signal bandwidth and to reduce the power consumption at the internal node (transmitter), the proposed system exploits the non-linearity of the metallic channel to transmit at higher resonance frequencies. Moreover, power consumption at the external node (receiver) is reduced by using a subsampling based receiver, which allows its implementation by low-cost electronics.
\end{abstract}

Index Terms - Intelligent Containers, Intermodal Transport, Metallic Channels, OFDM, Piezoelectric Transducers, Subsapling Receivers, Tracking, Ultrasonic Communication

\section{INTRODUCTION}

$\mathrm{R}$ esearch in ultrasonic communication has grown in recent years, especially for applications where sealing and structural integrity must be preserved, such as aeronautics and aerospace industry, and hermetically sealed tanks and fuselages [1]-[6]. For these applications, a wired communication proves unfeasible and even the effectiveness of a wireless communication is reduced in these harsh environments.

The system proposed in this paper is particularly oriented to cargo containers, which need to implement a continuous noninvasive inspection in order to monitor and report the state of the assets. The implementation of an efficient cargo monitoring system is fundamental due to the high impact of the freight transport on the global economy [7], specially the maritime freight transport, which represents the $75 \%$ of international freight transportation [8]. Therefore, there is an ongoing trend

Manuscript received February 19, 2018; revised August 29, 2018; accepted November 14, 2018. This work was supported by the Spanish Ministry of Economy and Competitiveness under Projects TEC2016-80396-C2-2-R and TEC2016-80396-C2-1-R. The Associate Editor for this article was X. Cheng. (Corresponding author: José R. García Oya.) to integrate Information and Communication Technologies (ICTs) with transportation systems [9].

The proposed system is based on placing several sensors inside the container, whose output data are collected by a microprocessor. This microprocessor transmits the information by an ultrasonic communication link through the metallic wall of the cargo container. Outside the containers, information is transmitted by a wireless network to an internet-gateway to manage this tracking and monitoring information by the end users of the logistics chain. The goal would be to approach to the paradigm of intelligent containers [10]-[11], so that all the stakeholders of the intermodal freight transport are interconnected. Since freight transport usually combines multiple modes (such as water, roads, railways), the intermodal transport framework proposes to improve this transportation simultaneously over all these specific modalities, implementing collaborative processes to integrate the possible influences of the different modes on the others [9], [12]. The end result will be to reduce delivery times and congestion, in order to decrease the impact on the environment, by implementing sustainable intelligent containers performing decisions locally to minimize the amount of data to be transmitted.

In [6] an experimentally validated system with tracking, security and management of goods purposes was presented. The main advantage of this system over other commercial solutions was the avoidance of any damage on the container's surface by using a non-intrusive technology based on ultrasonic communication. This paper is focused on improving the design of the ultrasonic front end, performing a more exhaustive study of the metallic channel in order to exploit its non-linearity to transmit at higher resonance frequencies. This will improve such previous work in terms of robustness, data rate and power consumption, increasing the battery life. The improvements achieved directly impact on the tracking and monitoring capabilities of the proposed intermodal freight transportation system.

J. R. García Oya, J. García Doblado, F. Muñoz Chavero, E. Hidalgo Fort, and V. Baena Lecuyer are with the Electronics Eng. Department, University of Seville, 41004 Seville, Spain (e-mail: jose.garciaoya @ gie.esi.us.es).

J. M. Algueta Miguel and A. J. López Martín are with the Institute of Smart Cities, Public University of Navarra, 31006 Pamplona, Spain.

Digital Object Identifier 10.1109/TITS.2018.2882565 
The paper is organized as follows. Section II summarizes several related works with applications in monitoring of cargo containers. Section III presents an overview of the whole system. Ultrasonic channel characterization is detailed in Section IV. From the obtained results, an enhanced ultrasonic front-end topology is proposed in Section V. Section VI describes the experimental results and Section VII presents a comparative study of similar research works. Finally, conclusions are drawn in Section VIII.

\section{RELATED WORKS}

The main concepts and benefits of automatizing the containers terminals were originally evaluated in [13]. By using the basis explored in [13], more recent research works have studied how to optimize the logistics algorithms to improve location, tracking and planning for roads [12], trains [14] and specially container seaports [15]-[16] applications. This interest is due to the fact that these maritime terminals represent the main interface and iteration point between the different freight transportation modalities. However, all these approaches do not provide an integrated solution with monitoring of goods functionalities. Besides, there are several complete solutions at research [17] and commercial [18] levels. However, these solutions present some drawbacks, such as using wireless communication at $2.4 \mathrm{GHz}$ (leading to communication issues due to the high density of stacked metal containers) and the use of invasive solutions, which need to drill the container surface to collect the sensed variables.

In order to overcome these limitations, [6] proposed, respectively, the use of both a low power wireless network at $868 / 915 \mathrm{MHz}$ and ultrasonic communication through the container metallic walls. This solution was selected over other non-invasive technologies, such as RFID, which presents collision and electromagnetic propagation problems for harsh scenarios with many stacked containers, and also reduced efficiency for monitoring and location purposes [19]. Other discarded non-invasive solutions are based on Optical Character Recognition (OCR) [20] and computer vision-based techniques [21]. These techniques imply more complex and error-prone processes, due to the high dependence with the properties of the container surface, which can be deteriorated during the transport, and also with conditions such as angle of view, distance or luminosity.

Finally, a numerical comparison between other related works based on ultrasonic communication through metal barriers, for low data rate [1]-[3], [6] and high data rate applications [4]-[5], will be detailed in Section V. More specifically, as mentioned above this paper presents an enhanced version of the previous work [6], focused on overcoming some limitations regarding power consumption, robustness and signal bandwidth. The main novelties of the proposed system regarding [6] can be summarized as follows:

a) Power consumption is reduced considerably by using a new transducer-metal coupling method more robust against fluctuations and with higher signal bandwidth and sensitivity, allowing reduction of the voltage generated at the input of the ultrasonic transducer at the transmitter side. b) The proposed transmitter implementation exploits the non-linearity of the metallic channel, by transmitting a $5^{\text {th }}$ harmonic over the operation frequency of the ultrasonic transducer. This innovative solution reduces the power consumption in the transmitter, which is a critical requirement for devices installed inside the container.

c) Power consumption is also reduced in the receiver side by implementing a subsampling based process for non-linear scenarios, which allows the use of low-cost electronics for higher data rates. Also, the employed carrier and sampling frequencies have been properly selected in order to avoid the use of a Direct Digital Synthesizer (DDS), reducing the complexity at the transmitter and receiver sides.

d) A more reliable modulation scheme (128-OFDM instead of DBPSK) is proposed in order to exploit the observed higher signal bandwidth. This multi-carrier modulation reduces the transmission times, improves robustness against fading effects and fluctuations of carrier frequency, and increases the spectrum efficiency. This latter advantage would allow higher data rates, extending the sensor capabilities. For example, by increasing the number of measurements, or including high data rate information.

\section{SYSTEM OVERVIEW}

The proposed overall system is composed of:

- Sensors inside the container.

- Ultrasonic communication link to extract information from the sensor handler to the transponder controller, both based on low power microprocessors.

- A wireless network to transmit the information between the containers.

- The internet-gateway and dedicated software to manage the information. This information will be presented to the final user and it will be used for tracking and monitoring purposes.

The features and functionalities of each part were detailed in [6] and can be identified in Figure 1. The system, based on noninvasive communication and portable devices, has a reduced cost-factor of design, manufacturing and maintenance, having a scalable price of $200-300 €$ per container, almost irrelevant compared with the investment for a container and the associated shipment costs.

The system was designed to work properly on a harsh environment based on stacked metallic containers, by selecting the frequency band 868/915 MHz (IEEE 802.15.4 standard), with worldwide compatibility, high information bandwidth and oriented to low power and efficient sensor networks. The proposed solution optimizes signal bandwidth and power consumption, by redesigning the ultrasonic communication link, as will be described in the next sections.

Figure 1. System overview

(C) 2018 IEEE. Personal use of this material is permitted. Permission from IEEE must be obtained for all other uses, in any current or future media, including reprinting/republishing this material for advertising or promotional purposes, creating new collective works, for resale or redistribution to servers or lists, or reuse of any copyrighted component of this work in other work. 


\section{Ultrasonic ChANNEL DESCRIPTION}

\section{A. Ultrasonic Technology Selection}

Piezoelectric transducers have been selected over other technologies, such as laser and electromagnetic acoustic transducers (EMAT), due to their higher maturity, transducers size, power efficiency, lower maintenance costs and higher data rates [22]. The main drawback associated to the piezoelectric techniques is the induced multipath propagation, leading to Inter-Symbol Interference (ISI). This limitation will be minimized, using a multi-carrier OFDM modulation, by selecting a proper cyclic prefix, as described in Section V. This allows increasing the carrier frequency and exploiting the benefits of these modulations regarding spectrum efficiency. The employed piezoelectric transducer is the model Prowave 400EP250 model, with a carrier frequency at $40 \mathrm{KHz}$.

\section{B. Transducer-Metal Coupling Selection}

The performance of the ultrasonic channel depends to a large extent on the quality of the coupling between the metallic wall and the ultrasonic transducer. In the previous system proposed in [6], a magnetic enclosure was employed to attach the transducers to the container wall, allowing an easy and versatile installation. However, this type of coupling can reduce the stability of the channel with respect to the frequency response, due to a lower acoustic impedance matching caused by insufficient pressure or surface roughness.

Other coupling methods based on permanent adhesives have been widely used in the literature [3], providing higher physical resistance and improved stability of the ultrasonic channel, at the expense of a more tedious installation.

In this context, both coupling approaches, i.e., the magnetic enclosures employed in [6] and the fixation using an adhesive (Araldite 2011 Epoxy), have been experimentally compared. A painted steel plate with an area of $1 \mathrm{~m}^{2}$ and a thickness of 0.7 $\mathrm{cm}$ has been employed for emulating the properties of the real cargo container in this preliminary test, placing the aligned transducers at both sides of the plate.

The main conclusion was the strong dependence of the frequency response with the coupling method used. The experimental results are shown in Figure 2. As can be observed, the transducers fixed with epoxy clearly offer a higher sensitivity, except for a narrow band around the nominal operating frequency $(40 \mathrm{KHz})$, where the system using magnetic cases presents its higher resonance peak. Moreover, it has been verified that the magnitude of this peak presents a slight variation (between $40-45 \mathrm{KHz}$ ) for different experiments. This drift is due to a reduction of acoustic impedance matching. In contrast, the transducers attached with epoxy offer a higher stability in a wide bandwidth, especially at higher frequencies, being the maximum sensitivity at around $360 \mathrm{KHz}$.

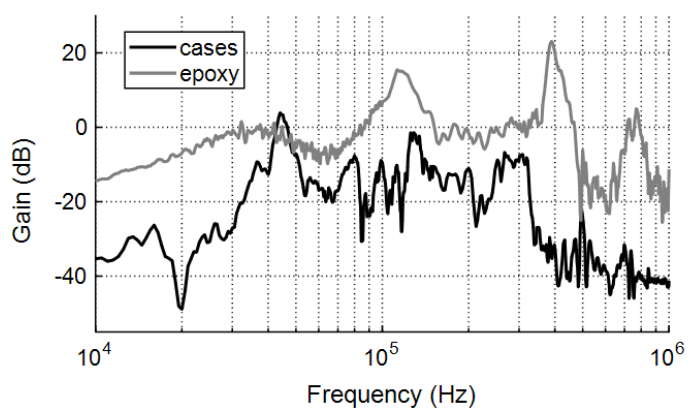

Figure 2. Frequency response using epoxy and magnetic cases

Additionally, note that the maximum of the transmitted ultrasonic wave is located at [2]:

$$
N=\left(D^{2}-\lambda^{2}\right) / 4 \lambda
$$

where $D$ is the diameter of the transducer $(25 \mathrm{~mm})$ and $\lambda=v / f$ is the wavelength, with the acoustic propagation velocity $\nu \approx 5150 \mathrm{~m} / \mathrm{s}$ for steel and $f=360 \mathrm{KHz}$. Thus, $N$ results $0.735 \mathrm{~cm}$, very similar to the metallic barrier thickness $(0.7 \mathrm{~cm})$, so the transmitter will work close to the optimal case. Therefore, the solution adopted to implement the final system will be based on epoxy coupling methods, instead of the magnetic cases used in [6], in order to exploit some features such as:

- A minimal frequency variation.

- High gain, up to $20 \mathrm{~dB}$ higher than working at the nominal resonance frequency using magnetic cases.

- Higher signal bandwidth (around $30 \mathrm{KHz}$ ) than working at the nominal resonance frequency $(10 \mathrm{KHz})$ [6].

Although using low frequencies has some benefits regarding insertion losses and robustness against multi-path effects, the advantages found by transmitting around the $360 \mathrm{KHz}$ band will lead to improve the overall performance of the communication system, as described in Section V.

\section{COMMUNICATION SYSTEM BASED ON OFDM}

\section{A. Modulation parameters and employed devices}

An OFDM modulation is implemented in order to exploit the higher bandwidth observed in the channel and provide additional robustness to the communication link. The implemented OFDM modulation is a simplified version of the standard PRIME v1.3.6 (PoweR line Intelligent Metering Evolution, standard developed by Power Line Communication).

. The main modulation parameters are the following:

- 128 carriers (97 active carriers).

- Possible modulations: DBPSK, DQPSK and D8PSK.

- Optional convolutional FEC.

- Cyclic prefix of 16 samples.

- Preamble for detection and synchronization purposes based on a chirp of 40 samples.

- $\quad$ Signal bandwidth of $24.5 \mathrm{kHz}$.

- Separation between the carriers of $250 \mathrm{~Hz}$.

In order to reduce the power consumption, modulation and demodulation processes are implemented by software using two 
low-cost microcontrollers 32bit-ARM Cortex-M3 STM32F103RD. With the same objective, the 12-bit DAC and ADC embedded in the microcontroller have been employed.

\section{B. Transmitter implementation}

A diagram of the implemented OFDM system is shown in Figure 3.The transmitter (TX) encodes a binary data stream to generate discrete-time OFDM symbols, using a convolutional FEC (Forward Error Correction) with code generator polynomials equal to 1111001 and 1011011. This FEC can be disabled in order to increase the overall bitrate. After coding, the binary stream is randomized by the scrambler block, reducing the crest factor when there is a long stream of ' 0 's or ' 1 's. Due to frequency fading, OFDM subcarriers are usually received with different amplitudes and, therefore, some groups of subcarriers can be less reliable than others, causing more bit errors in bursts if they are not randomly scattered. Moreover, the interleaver permutes the order of bits to ensure that originally adjacent bits are separated by several bits.

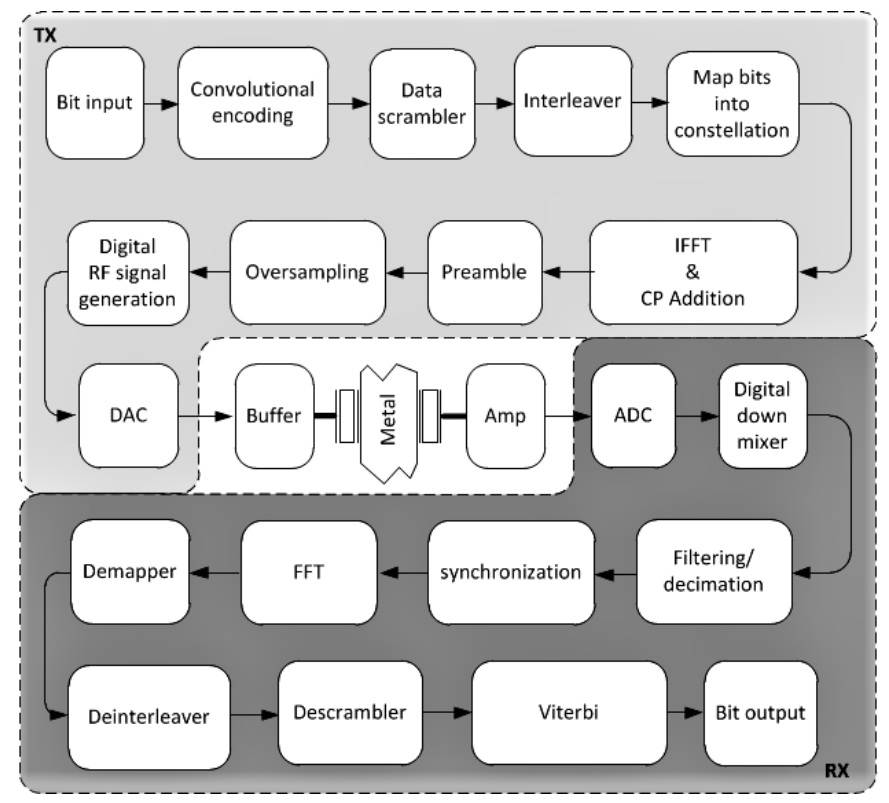

Figure 3. Block diagram of the OFDM system

After the interleaving process, the binary data is modulated as a multi-carrier DPSK signal with a pilot and 128 data subcarriers allowing the transmission of 128, 256 or 512 bits per symbol. The frequency domain OFDM symbol is converted to time domain by means of an IFFT process and the cyclic prefix is added to cancel the multipath effect. In order to carry out the synchronization process at the receiver side, a preamble is added at the beginning of the transmission frame. After that, the signal is up-converted to $72 \mathrm{KHz}$ and then converted to the analog domain by using a 12-bit DAC.

This carrier frequency $\left(f_{c l}\right)$ of $72 \mathrm{KHz}$ has been selected with the objective to generate the signal directly, without using an external quadrature mixer, thus reducing costs. Thus, we select a DAC sampling frequency $\left(f_{1}\right)$ of $288 \mathrm{KHz}$, i.e., $f_{s l}=4 \bullet f_{c l}$. This frequency plan allows the immediate implementation of a digital mixer, without implementing a DDS, i.e., only using values of \pm 1 and 0 for the mixing process. Moreover, by selecting this value of $f_{c l}$, it is possible to exploit the high non- linearity of the channel to transmit a signal located at $360 \mathrm{KHz}$, using the $5^{\text {th }}$ harmonic of the generated signal. This solution allows to transmit within the band of highest gain for the case of epoxy attachment (Figure 2), maintaining the use of a low cost DAC embedded in the microcontroller. Finally, a buffer is employed to drive the ultrasonic transducer.

\section{Receiver implementation}

\section{1) Charge amplifier stage}

This stage performs the charge-voltage conversion of the ultrasonic signal, as was described in [6], and it is based on an operational amplifier (OPA350 of Texas Instruments) with a negative feedback loop composed by a capacitor $\left(C_{f}\right)$ and a resistor $\left(R_{f}\right)$, leading to a $1^{\text {st }}$-order high pass response, with a cutoff frequency at $1 /\left(2 \pi C_{f} R_{f}\right)$ and a sensitivity inversely proportional to $C_{f}$. A value of $10 \mathrm{pF}$ for $C_{f}$ and a $R_{f}$ higher than $50 \mathrm{~K} \Omega$ have been selected in order to maximize the sensitivity and to place the band of interest (i.e., a signal centered at 360 $\mathrm{KHz}$ with $24.5 \mathrm{KHz}$ of signal bandwidth) in the pass-band of the amplifier.

\section{2) Analog-to-digital conversion stage}

For this application we propose to implement a subsampling based receiver, in order to reduce the power consumption at the external node and to implement a detection process portable to lower cost ADCs. For a bandpass signal centered at $f_{c}$ with a signal bandwidth $B$, the subsampling frequency $\left(f_{s}\right)$ has to meet Equation 2 [23], in order to avoid aliasing between the replicas folded in the spectrum:

$$
2\left(f_{c}-B / 2\right) /(m-1)>f_{s}>2\left(f_{c}+B / 2\right) / m
$$

where $m$ is the number of replicas of the signal in the range $\left[0, f_{c}-B / 2\right]$, with a value between 1 and floor $\left(\left(f_{c}+B / 2\right) / B\right)$. Additionally, for a nonlinear environment, as the described ultrasonic metal channel, there are additional challenges to avoid aliasing between the different harmonics. For these scenarios, the selected sampling frequency has to meet this additional requirement [24]:

$$
\text { if } f_{c}+n_{k} f_{s} \leq j f_{c}<i f_{c}+\left(n_{k}+1\right) f_{s}
$$

where $i f_{c}$ and $j f_{c}$ are two harmonics of $f_{c}$ and $n_{k}=$ floor $\left(\left(j f_{c}-\right.\right.$ $\left.i f_{\mathrm{c}}\right) / f_{s}$ ). Since this implementation is a particular case, working with the $5^{\text {th }}$ harmonic as signal of interest $\left(f_{c}\right)$, we assign to $i$ and $j$ values multiples of $1 / 5$ instead of integer values, avoiding aliasing between the $5^{\text {th }}$ harmonic and the original signal at 72 $\mathrm{KHz}$ and its $2^{\text {nd }}-4^{\text {th }}$ and $6^{\text {th }}$ harmonics. $A 6^{\text {th }}$ order non-linearity has been assumed since the channel response attenuates around $40 \mathrm{~dB}$ the $>6^{\text {th }}$ order harmonics, as shown in Figure 2.

Between the valid sampling ranges given by (2)-(3), sampling frequency $\left(f_{s 2}\right)$ of $320 \mathrm{KHz}$ is selected, so that $f_{c l}$ (at $360 \mathrm{KHz})$ is folded to $40 \mathrm{KHz}\left(f_{c 2}\right)$. Therefore, the digital mixing process is implemented by ADC sampling, reducing the used resources. Since the sampling frequency is $f_{s 2}=8 \bullet f_{c 2}$, it is not necessary to use a DDS, due the samples of the discrete sine and cosine only require the values of $\pm 1, \pm 1 / \sqrt{ } 2$ and 0 .

\section{3) Digital domain stage}

In the digital domain the signal is down-converted, filtered and decimated, as illustrated in Figure 3, in order to obtain the baseband complex OFDM symbols. Time synchronization is carried out using a simple correlation with the received signal and the preamble. Next, the cyclic prefix, which can be corrupted by the multipath echoing, is removed. Each time- 
domain OFDM symbol is processed by the FFT block, recovering the magnitude and phase of each OFDM subcarrier. Then, the data carriers are demapped, deinterleaved and descrambled, and finally decoded using a Viterbi decoder.

\section{EXPERIMENTAL RESULTS}

For this test, the transducers were fixed to the metallic wall, faced at both sides of the cargo container (with a thickness of 7 $\mathrm{mm}$ and dimensions according to the International Standards Organization (ISO) [19]), as illustrated in Figure 4.

With the objective to decide the optimal modulation in terms of BER and energy efficiency, a comparison between three modulations, with five different voltage levels at the DAC output, and with or without using a FEC, is presented. For each experimental measurement, 2000 data bursts, with length of 33 bytes, are transmitted. The results are shown in Table I, where it is possible to observe the expected performance, i.e., a higher robustness when a FEC and a higher input voltage are employed. However, even for a minimal input voltage, the obtained BER results satisfactory.

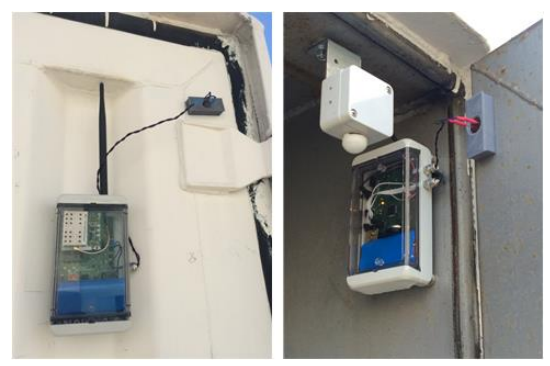

Figure 4. Transponder controller outside the container (left) and acquisition board inside the container (right)

Additionally, the energy consumption has been evaluated for the same 30 scenarios. These results are presented in Table II, showing the energy consumed by data burst (33 bytes), assuming a normalized resistance of $1 \Omega$. The transmission time can be expressed as:

$$
T_{t x}=\left(N_{p r}+\left(N_{c p}+N_{i f f t}\right) N_{s y m}\right) / f_{b b}
$$

where $N_{p r}, N_{c p}, N_{i f f t}$ are, respectively, the number of samples per preamble (40), per cyclic prefix (16) and per symbol (128), $N_{s y m}$ the number of symbols necessary to transmit 33 bytes for each modulation, and $f_{b b}$ the baseband sampling frequency ( 32 $\mathrm{KHz})$.

TABLE I

BIT ERROR RATE FOR THE DIFFERENT SCENARIOS

\begin{tabular}{cccccc}
\hline \hline & $\mathbf{5 0}$ & $\mathbf{2 5}$ & $\mathbf{1 2}$ & $\mathbf{7}$ & $\mathbf{4}$ \\
DBPSK & $\mathbf{m} \mathbf{V}_{\text {rms }}$ & $\mathbf{m} \mathbf{V}_{\text {rms }}$ & $\mathbf{m V}_{\text {rms }}$ & $\mathbf{m V}_{\text {rms }}$ & $\mathbf{m V}_{\text {rms }}$ \\
DQPSK & $0^{1}$ & 0 & 0 & 0 & 0.00007 \\
D8PSK & 0 & 0 & 0 & 0.00024 & 0.0149 \\
DBPSK+FEC & 0 & 0.0003 & 0.003 & 0.02040 & 0.0718 \\
DQPSK+FEC & 0 & 0 & 0 & 0 & 0 \\
D8PSK+FEC & 0 & 0 & 0 & 0 & 0 \\
\hline \hline
\end{tabular}

'BER $=0$ ' strictly equals to $\mathrm{BER}<2 \cdot 10^{-5}$ (in order to implement more reliable measurements, the error condition is given by receiving at least 10 wrong bits, i.e., the resolution is 10 errors/(2000 data bursts $\bullet 33$ bytes $\bullet 8$ bits $)$ ).
NORMALIZED ENERGY CONSUMPTION $[\mu \mathrm{J}]$ FOR THE DIFFERENT SCENARIOS

\begin{tabular}{cccccc}
\hline \hline & $\mathbf{5 0}$ & $\mathbf{2 5}$ & $\mathbf{1 2}$ & $\mathbf{7}$ & $\mathbf{4}$ \\
& $\mathbf{m} \mathbf{V}_{\text {rms }}$ & $\mathbf{m V}_{\text {rms }}$ & $\mathbf{m V}_{\text {rms }}$ & $\mathbf{m V}_{\text {rms }}$ & $\mathbf{m V}_{\text {rms }}$ \\
DBPSK & 36.875 & 9.21875 & 2.124 & 0.72275 & 0.236 \\
DQPSK & 25.625 & 6.40625 & 1.476 & 0.50225 & 0.164 \\
D8PSK & 14.375 & 3.59375 & 0.828 & 0.28175 & 0.092 \\
DBPSK+FEC & 70.625 & 17.65625 & 4.068 & 1.38425 & 0.452 \\
DQPSK+FEC & 36.875 & 9.21875 & 2.124 & 0.72275 & 0.236 \\
D8PSK+FEC & 25.625 & 6.40625 & 1.476 & 0.50 .225 & 0.164 \\
\hline \hline
\end{tabular}

Moreover, EVM (Error Vector Magnitude) has been calculated and is shown in Table III. This figure of merit, which is independent of modulation, measures how far the constellation points are from the ideal locations, and is mathematically given by (5) [25]:

$$
E V M=\frac{\sum_{i=1}^{L} \sum_{k=2}^{65}\left|r_{k}^{i}-r_{k-1}^{i} e^{-(j 2 \pi / M) \Delta b_{k-1}}\right|^{2}}{\sum_{i=1}^{L} \sum_{k=2}^{65}\left|r_{k}^{i}\right|^{2}}
$$

where $L$ is the number of OFDM symbols, $r_{k}^{i}$ denotes the $k^{\text {th }}$ carrier of the FFT output for symbol $i . \Delta b_{k}$ represents the decision on the received information symbol coded in phase increment, and $M$ is the number of constellation symbols, i.e., 2,4 , or 8 for DBPSK, DQPSK or D8PSK, respectively.

TABLE III

EVM FOR THE DIFFERENT SCENARIOS

\begin{tabular}{cccccc}
\hline \hline & $\mathbf{5 0}$ & $\mathbf{2 5}$ & $\mathbf{1 2}$ & $\mathbf{7}$ & $\mathbf{4}$ \\
& $\mathbf{m} \mathbf{V}_{\text {rms }}$ & $\mathbf{m V}_{\text {rms }}$ & $\mathbf{m V}_{\text {rms }}$ & $\mathbf{m V}_{\text {rms }}$ & $\mathbf{m V}_{\text {rms }}$ \\
DBPSK & 19.8 & 18.86 & 16.51 & 12.56 & 7.78 \\
DQPSK & 19.24 & 18.63 & 16.12 & 12.25 & 7.49 \\
D8PSK & 19.42 & 18.68 & 16.14 & 12.29 & 8.83 \\
DBPSK+FEC & 19.16 & 18.38 & 16.24 & 12.3 & 7.64 \\
DQPSK+FEC & 18.95 & 18.29 & 16.27 & 12.31 & 7.82 \\
D8PSK+FEC & 19,15 & 18.5 & 16.24 & 12.42 & 8.78 \\
\hline \hline
\end{tabular}

From this analysis, it is possible to observe how the optimal modulation in terms of accuracy results on a D8PSK (at 50 $\mathrm{mV}_{\mathrm{rms}}$ ), since it minimizes the consumed energy between scenarios with minimum BER and maximum EVM. For cases where the energy consumption is the main requirement, the best option correspond to a DQPSK with the activated FEC (at 4 $\mathrm{mV}_{\mathrm{rms}}$ ), considering the scenarios with minimal BER.

Figure 5 (left) illustrates the constellation measured for a D8PSK, where it is possible to observe how the locations between the eight regions are sufficiently separated. Figure 5 (right) shows the 256 points-FFT of the channel response. It is possible to observe how, for the band of interest (59.75-84.25 $\mathrm{KHz}$ ), is very close to the maximum gain region, with a gain ripple of 58 points, i.e., $1.16 \mathrm{~dB}$.
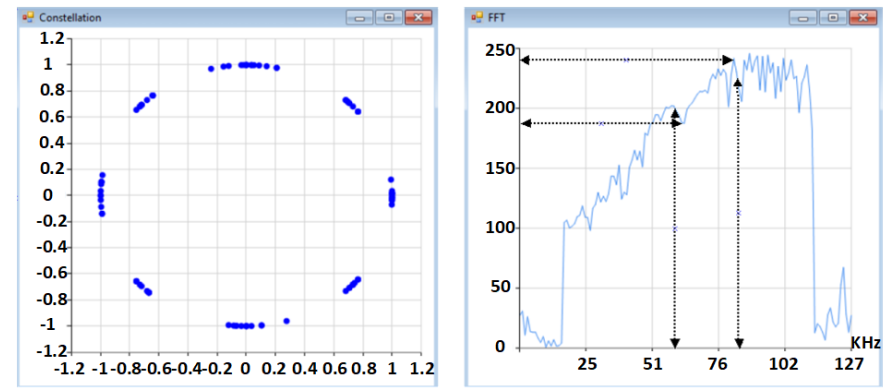

Figure 5. Constellation (left) and channel response (right) for a D8PSK 


\section{COMPARATIVE ANALYSIS}

A comparative study of ultrasonic systems through metal walls is shown in Table IV, which includes the features of the proposed system and the previous version presented in [6].

In this table, only [4] uses EMAT instead of piezoelectric technologies, presenting a lower power efficiency, typical of EMAT technologies. Additionally, although [4]-[5] provides a high data rate performance, they also present higher power consumption since the attenuation through the metal channel increases with the frequency. This higher power consumption is also due to they use thicker metallic barriers, as the case presented in [1]. For low power applications, such as [2]-[3], the proposed system considerably improves the power consumption and the data rate, by using an OFDM instead of an AM modulation, leading to enhanced features regarding transmission times and spectrum efficiency.

Furthermore, it is possible to observe how the proposed system significantly improves the previous version [6]. Firstly, by using the resonance band at $360 \mathrm{KHz}$, it is possible to increase the signal bandwidth up to $24.5 \mathrm{KHz}$, which leads to a higher data rate $(50.6 \mathrm{Kbps})$ and a lower transmission time. This time per data burst is minimum (5.75 ms from equation (4)) for the D8PSK case and maximum $(28.25 \mathrm{~ms})$ for the DBPSK+FEC, in contrast with the transmission time of 108.8 ms obtained in [6]. Secondly, the proposed system considerably reduces the power consumption (calculated for a $300 \Omega$ transducer input impedance), by exploiting the gain in the selected band, which is around $20 \mathrm{~dB}$ higher than the gain at 40 $\mathrm{KHz}$ by using magnetic cases. This power consumption is given for the scenario with higher EVM (input voltage of $50 \mathrm{mV}_{\mathrm{rms}}$ ), being still possible to reduce this voltage keeping a good performance in terms of BER. Besides exploiting a higher gain, it is possible to reduce this input voltage since the proposed system presents an enhanced robustness against frequency drift and fading effects, by using an epoxy based coupling method and a multi-carrier modulation, respectively.

TABLE IV

COMPARISON BETWEEN DIFFERENT RELATED WORKS BASED ON ULTRASONIC TRANSMISSION

\begin{tabular}{ccccc}
\hline \hline Publication & $\begin{array}{c}\text { Data rate } \\
\text { (Kbps) }\end{array}$ & Modulation & $\begin{array}{c}\text { Voltage / } \\
\text { Power Input }\end{array}$ & $\begin{array}{c}\text { Bulkhead } \\
\text { Thickness } \\
\text { (cm) }\end{array}$ \\
{$[1]$} & 0.435 & AM & $10 \mathrm{~V}$ & 15.24 \\
{$[2]$} & 1 & AM & $30 \mathrm{~mW}$ & 0.7 \\
{$[3]$} & 32.5 & AM & $100 \mathrm{~mW}$ & 5.7 \\
{$[4]$} & 1000 & QPSK & $1230 \mathrm{~mW}$ & 2.54 \\
{$[5]$} & 17370 & OFDM & $31 \mathrm{~V}$ & 6.35 \\
{$[6]$} & 2.5 & DBPSK & $3.3 \mathrm{~V} / 36.3 \mathrm{~mW}$ & 1 \\
This work & 50.6 & OFDM & $141.1 \mathrm{mV} / 66.7 \mu \mathrm{W}$ & 0.7 \\
\hline \hline
\end{tabular}

This enhanced robustness can also be evidenced by inspecting the reliability of the whole system. Once the performance of the sensors inside the container (i.e., temperature, brightness, humidity, accelerometer, motion and magnetic), the wireless network, the GPS and the server and data browser were validated as described in [6], the integrated system led to a $99.47 \%$ of correct measurements, improving the $97.33 \%$ value in [6] for the same number of measurements and three cargo containers monitored simultaneously.

\section{CONCLUSIONS}

A low-cost and non-invasive ultrasonic communication based system has been presented for tracking, monitoring and management of cargo containers, where the sealing preservation and the reduction of the power consumption are the main technical challenges. The proposed system employs an OFDM modulation with the objective to increase the data rate and reduce the transmission times. The system uses an epoxy based coupling method in order to improve the robustness against drift of the carrier frequency and to transmit over the nominal frequency of the piezoelectric transducer, increasing the signal bandwidth and the gain at the selected band. As main novelty, we propose to exploit this higher gain and the nonlinearity of the channel, transmitting the $5^{\text {th }}$ harmonic of the generated signal, making it possible to reduce the power consumption at the internal node. At the receiver side, a subsampling based system allows to reduce power consumption of the external node. Thanks to the subsampling process, the proposed communication system can be implemented using low-cost and low power electronics. A comparison of different angular modulation has been performed in terms of BER and consumed energy. The designed communication system has been integrated in the whole system, composed of an internal configurable sensor network, a long range and low power scalable wireless IEEE 802.15.4 network at $868 / 915 \mathrm{MHz}$, with worldwide compatibility and oriented to harsh scenarios, and a management subsystem for tracking and monitoring purposes. The integrated system has been experimentally validated in a real scenario, leading to a competitive solution for intermodal freight transport, and improving the features of the previous work regarding power consumption, data rate and robustness.

\section{REFERENCES}

[1] G. J. Saulnier et al., "Through-Wall Communication of Low-Rate Digital Data Using Ultrasound," IEEE Ultrason. Symp., pp. 1385-1389, Oct. 2006.

[2] M. Kluge et al., "Remote Acoustic Powering and Data Transmission for Sensors inside of Conductive Envelopes," IEEE Sensors, pp. 41-44, Oct. 2008.

[3] J. D. Ashdown, et al., "A Full-Duplex Ultrasonic Though-Wall Communication and Power Delivery System," IEEE Trans. on Ultrason., Ferroelectr., and Freq. Control, vol. 60, no. 3, March 2013.

[4] D. J. Graham, J. A. Neasham and B. S. Sharif, "Investigation of Methods for Data Communication and Power Delivery Through Metals," IEEE Trans. on Ind. Elecron., vol. 58, no. 10, pp. 4972-4980, Oct. 2011.

[5] T. J. Lawry et al., "A High-Performance Ultrasonic System for the Simultaneous Transmission of Data and Power Through Solid Metal Barriers," IEEE Trans. on Ultrason., Ferroelectr., and Freq. Control, vol. 60, no. 1, pp. 194-205, Jan. 2013.

[6] E. H. Fort et al., "Intelligent Containers Based on a Low-Power Sensor Network and a Non-Invasive Acquisition System for Management and Tracking of Goods," IEEE Trans. on Intell. Transp. Sys., vol. PP, no. 99, pp. 1-6, 2017.

[7] F. Corman et al., "Optimizing Hybrid Operations at Large-Scale Automated Container Terminals," Models and Technologies for Intelligent Transportation Systems (MT-ITS), pp. 514-521, Jun. 2015.

[8] E. Bou-Harb, E. I. Kaisar and M. Austin, "On the Impact of Empirical Attack Models Targeting Marine Transportation," 5th IEEE Intern. Conf. on Models and Technologies for Intell. Transp. Sys. (MT-ITS), pp. 200205, 2017.

[9] G. Cavone, M. Dotoli and C.Seatzu, "A Survey on Petri Net Models for Freight Logistics and Transportation Systems," IEEE Trans. on Intell. Transp. Syst., vol. PP, no. 99, pp. 1-19, 2017. 
[10] W. Lang et al., "The "Intelligent Container"-A Cognitive Sensor Network for Transport Management," IEEE Sensors Journal, vol. 11, no.3, pp. 688-698, Mar. 2011.

[11] R. Jedermann, C. Lloyd and T. Pötsch, "Communication techniques and challenges for wireless food quality monitoring," Philosophical Transactions of the Royal Society A, vol. 372, no. 2017, pp. 1-18, 2017.

[12] A. Abadi, P. A. Ioannou and M. M. Dessouky, "Multimodal Dynamic Freight Load Balancing," IEEE Trans. on Intell. Transp. Syst., vol. 17, no. 2, pp. 356-366, Feb. 2016.

[13] C. I. Liu, H. Jula and P. A. Ioannou, "Design, Simulation, and Evaluation of Automated Containers Terminals," IEEE Trans. on Intell. Transp. Sys., vol. 3, no. 1, pp. 12-26, Mar. 2002.

[14] W. Sun et al., "Energy-Efficient Communication-Based Train Control Systems With Packet Delay and Loss," IEEE Trans. on Intell. Transp. Syst., vol. 17, no. 2, pp. 452-468, Feb. 2016.

[15] C. Caballini et al., "An Event-Triggered Receding-Horizon Scheme for Planning Rail Operations in Maritime Terminals," IEEE Trans. on Intell. Transp. Syst., vol. 15, no. 1, pp. 365-375, Feb. 2014.

[16] L. Chen et al., "Container Port Performance Measurement and Comparison Leveraging Ship GPS Traces and Maritime Open Data," IEEE Trans. on Intell. Transp. Syst., vol. 17, no. 5, pp. 1227-1242, May. 2016.

[17] S. Mahlknecht and S. A. Madani, "On Architecture of Low Power Wireless Sensor Networks for Container Tracking and Monitoring
Applications," $5^{\text {th }}$ IEEE Int. Conf. on Ind. Informat., vol. 1, no. 1, pp. 353358, June 2007.

[18] http://www.globetracker.com/gt-sense-genset/

[19] S. Abbate et al., "An Integer Linear Programming Approach for RadioBased Localization of Shipping Containers in the Presence of Incomplete Proximity Information," IEEE Trans. on Intell. Transp. Syst., vol. 13, no. 3, pp. 1404-1419, Sep. 2012.

[20] O. Bulan et al., "Segmentation- and Annotation-Free License Plate Recognition With Deep Localization and Failure Identification," IEEE Trans. on Intell. Transp. Syst., vol. 18, no. 9, pp. 2351-2363, Sep. 2017.

[21] X. Sun et al. , "A Generic Framework for Monitoring Local Freight Traffic Movements Using Computer Vision-based Techniques," 5th IEEE Intern. Conf. on Models and Technologies for Intell. Transp. Sys. (MTITS), pp. 63-68, 2017.

[22] R. A. Primerano, "High Bit-rate Digital Communication through Metal Channels," Ph.D. dissertation, Faculty of Drexel University, July 2010.

[23] R. Vaughan, N. Scott, and D. White, "The theory of bandpass sampling," IEEE Trans. Signal Process., vol. 39, no. 9, pp. 1973-1984, Sep. 1991.

[24] J. R. G. Oya et al., "Design of Dual-Band Multi-Standard Subsampling Receivers for Optimal SNDR in Nonlinear and Interfering Environments," IEEE Trans. on Instr. Meas., vol. 63, no. 4, pp. 981-983, Apr. 2014.

[25] "Narrowband orthogonal frequency division multiplexing power line communication transceivers for PRIME networks", ITU-T Rec.9904, Oct. 2012.

(C) 2018 IEEE. Personal use of this material is permitted. Permission from IEEE must be obtained for all other uses, in any current or future media, including reprinting/republishing this material for advertising or promotional purposes, creating new collective works, for resale or redistribution to servers or lists, or reuse of any copyrighted component of this work in other work. 